\title{
Factores asociados al ataque cerebrovascular isquémico entre los años 2013 a 2016: estudio de casos y controles
}

\author{
Juan-David Vega P. ${ }^{a, *}$, Álvaro-Andrés Ramos S. ${ }^{b}$, Edgar-Antonio Ibáñez P. ${ }^{a}$ \\ y Elisa-Andrea Cobo M. ${ }^{\text {a }}$
}

\author{
a Universidad de Boyacá, Tunja, Colombia \\ b Servicio de urgencias, Clínica Especializada Los Andes, Tunja, Colombia
}

Recibido el 20 de enero de 2017; aceptado el 2 de abril de 2017

Disponible en Internet el 6 de octubre de 2017

\section{PALABRAS CLAVE \\ Circulación \\ cerebrovascular; \\ Cerebro; \\ Factores de riesgo}

\begin{abstract}
Resumen
Objetivo: Determinar los factores asociados al ataque cerebrovascular isquémico en el servicio de urgencias de la Clínica Especializada Los Andes, de la ciudad de Tunja, entre los años 2013 a 2016.

Pacientes y métodos: Estudio de casos y controles; los casos correspondieron a 97 pacientes con ataque cerebrovascular isquémico (infarto cerebral isquémico y accidente isquémico transitorio) y los controles a 291 pacientes sin ataque cerebrovascular isquémico que ingresaron a urgencias entre los años 2013 a 2016.

Resultados: El sexo femenino correspondió al 56,7\% (55) de los casos y al 54,6\% de los controles (154) $(p=0,069)$. La media de edad en el grupo caso fue de 73,7 años [DE: 10,5 años] y en los controles de 64,5 años [DE: 11,3 años]. Los factores asociados al ataque cerebrovascular isquémico fueron: antecedente de ataque cerebrovascular isquémico [OR 7,7 IC 95\% 3,2; 18 $p=0,000$ ], tabaquismo [OR 4,4 IC 95\% 1,1; $18 \mathrm{p}=0$,022], dislipidemia [OR 3 IC 95\% 1,2; 7,5 p= 0,017 ], edad igual o mayor a 70 años [OR 2,3 IC $95 \% 1,3 ; 4,1 \mathrm{p}=0,002$ ] e hipertensión arterial [OR 1,8 IC 95\% 1,06; 3,3 p= 0,029].

Conclusiones: Los factores asociados al ataque cerebrovascular isquémico fueron, en orden de importancia, antecedente de ataque cerebrovascular isquémico, tabaquismo, dislipidemia, edad igual o mayor a 70 años e hipertensión arterial.

(C) 2017 Sociedad Colombiana de Cardiología y Cirugía Cardiovascular. Publicado por Elsevier España, S.L.U. Este es un artículo Open Access bajo la licencia CC BY-NC-ND (http:// creativecommons.org/licenses/by-nc-nd/4.0/).
\end{abstract}

\footnotetext{
* Autor para correspondencia.

Correo electrónico: ddavids89@hotmail.com (J.-D. Vega P.).
} 


\section{KEYWORDS}

Cerebrovascular

circulation;

Brain;

Risk factors
Factors associated with ischaemic cerebrovascular accidents between the years 2013 and 2016: A case-control study

\begin{abstract}
Objective: To determine the factors associated with ischaemic cerebrovascular accidents (ICVA) in the Emergency Department of the Andes Specialist Clinic of the city of Tunja, between the years 2013 and 2016.

Patients and methods: A case-control study was conducted in which the cases consisted of 97 patients with ICVA (ischaemic cerebral infarction and transient ischaemic accident), and the controls were 291 patients with no ICVA, who were admitted to the Emergency Department between the years 2013 and 2016.

Results: There were $56.7 \%$ (55) females in the cases, and $54.6 \%(154)$ in the controls $(P=.069)$. The mean age of the cases was 73.7 years [SD: 10.5 years], and 64.5 years [SD: 11.3 years] in the controls. The factors associated with ICVA were: a history of ICVA [OR; 7.7, 95\% Cl; 3.2-18, $P=.000$ ], smoking [OR; $4.4,95 \% \mathrm{Cl} ; 1.1-18, P=.022$ ], dyslipidaemia [OR; 3, 95\% Cl; 1.2-7.5, $P=.017$ ], age equal to or greater than 70 years [OR; $2.3,95 \% \mathrm{Cl} ; 1.3-4.1, P=.002$ ], and arterial hypertension [OR; $1.8,95 \% \mathrm{Cl} ; 1.06-3.3, P=.029]$.

Conclusions: The factors associated with ischaemic cerebrovascular accident were, in order of importance, a history of ischaemic cerebrovascular accident, smoking, dyslipidaemia, age equal to or greater than 70 years, and arterial hypertension.

(c) 2017 Sociedad Colombiana de Cardiología y Cirugía Cardiovascular. Published by Elsevier España, S.L.U. This is an open access article under the CC BY-NC-ND license (http:// creativecommons.org/licenses/by-nc-nd/4.0/).
\end{abstract}

\section{Introducción}

En el mundo, el ataque cerebrovascular es la segunda causa de mortalidad, a la que se le atribuyen 5.5 millones de muertes por año ${ }^{1}$ y es, además, la principal causa de discapacidad ${ }^{2}$. En Latinoamérica, las enfermedades cardiovasculares producen casi un millón de muertes, de las cuales el ataque cerebrovascular ocupa el segundo lugar con $28,3 \%^{3}$. En Colombia, la mortalidad por ataque cerebrovascular ocupa el cuarto lugar, y causó el $6,7 \%$ del total de muertes en el $2005^{4}$.

En Latinoamérica existen pocos estudios sobre ataque cerebrovascular isquémico ${ }^{5}$; respecto a Colombia y, en particular al departamento de Boyacá, no se han realizado investigaciones que permitan identificar la asociación con sus posibles factores de riesgo. Esta es la razón que justificó iniciar este proceso de investigación con un estudio observacional analítico y en una de las tres instituciones de salud más importantes de la capital del departamento de Boyacá. El objetivo principal fue determinar los factores asociados al ataque cerebrovascular isquémico en el servicio de urgencias de la Clínica Especializada Los Andes entre los años 2013 y 2016.

\section{Pacientes y métodos}

Se llevó a cabo un estudio observacional analítico de casos y controles en la clínica en mención. Se obtuvo información por medio de una ficha de recolección de datos tomados de los registros de historias clínicas de los pacientes que ingresaron al servicio de urgencias entre el primero de enero del año 2013 y el treinta de junio de 2016. La recolección de datos se hizo entre el 15 de junio y el 30 de agosto de 2016.
Los controles fueron seleccionados por medio de un muestreo probabilístico aleatorio simple y los casos por medio de los códigos CIE 10 que se muestran a continuación:

- 164: accidente vascular encefálico agudo, no especificado como hemorrágico o isquémico.

- 1632: infarto cerebral debido a oclusión o estenosis no especificada de arterias precerebrales.

- 1633: infarto cerebral debido a trombosis de arterias precerebrales.

- 1634: infarto cerebral debido a embolia de arterias cerebrales.

- 1635: infarto cerebral debido a oclusión o estenosis no especificada de arterias cerebrales.

- 1638: otros infartos cerebrales

- 1639: infarto cerebral no especificado.

- 1678: otras enfermedades cerebrovasculares especificadas.

- 1679: enfermedad cerebrovascular no especificada.

- 1688: otros trastornos cerebrovasculares en enfermedades clasificadas en otra parte.

- 1698: secuelas de otras enfermedades cerebrovasculares y de las no especificadas.

Los casos son pacientes con diagnóstico de ataque cerebrovascular isquémico (infarto cerebral isquémico confirmado por neuroimagen o accidente isquémico transitorio) con edad entre 50 y 90 años y que registraron ingreso al servicio de urgencias entre el $1 .^{\circ}$ de enero de 2013 y el 30 de junio de 2016. Los controles son pacientes sin diagnóstico de ataque cerebrovascular isquémico con edad entre 50 a 90 años que consultaron en el mismo periodo de tiempo. Los criterios de exclusión corresponden a pacientes 
con diagnóstico de trombosis cerebral venosa, tumores del sistema nervioso central, infecciones del sistema nervioso central, epilepsia, accidente cerebrovascular hemorrágico o hipoglucemia.

El cálculo del tamaño de la muestra se realizó con base en el estudio de Frometa ${ }^{6}$, del cual se seleccionó el factor estudiado de menor prevalencia (diabetes mellitus 18,5\%, OR: 2,67 ), nivel de confianza del $95 \%$, potencia del $80 \%$ y una relación de 3 controles por 1 caso, para una muestra de 97 casos y 291 controles, utilizando el programa Epidat versión 4.1. En este estudio la variable dependiente corresponde al ataque cerebrovascular isquémico y las variables independientes o predictores son: edad, sexo, índice de masa corporal, hipertensión arterial, diabetes mellitus, dislipidemia, tabaquismo, fibrilación auricular, antecedente de ataque cerebrovascular isquémico y antecedente de infarto agudo de miocardio; a continuación se dan a conocer las definiciones conceptuales, operativas y escala de medición de las variables (tabla 1 ).

Con respecto a los métodos estadísticos, la base de datos fue registrada en Excel versión 2013 y se analizó en el paquete estadístico SPSS versión 21. El análisis univariado se hizo por medio de estadística descriptiva a cada grupo (caso y control), determinando frecuencias absolutas y relativas en las variables categóricas; en el caso de las variables cuantitativas se calcularon medidas de tendencia central y de dispersión. El análisis bivariado se hizo para determinar las asociaciones posibles entre variables categóricas.

Se hicieron tablas tetracóricas con medición de $\mathrm{Chi}^{2}$ de Pearson y razón de disparidad (OR) con sus respectivos intervalos de confianza (IC) al 95\% (> 1 como factor de riesgo, $<1$ como factor protector y 1 sin asociación). También se determinó la diferencia de medias en las variables cuantitativas para grupo caso y control. Según la prueba estadística de Kolmogorov, si la variable cuantitativa presenta una distribución normal se utiliza la T de student y de no presentar normalidad se usa la $U$ de Mann-Whitney. En el análisis multivariado se realizó un modelo de regresión logística binaria con el método introducir, en el que se añadieron al modelo los factores estudiados que mostraron asociación en el análisis bivariado del estudio y aquellos con un valor representativo teórico.

En este diseño de investigación los sesgos a controlar pueden ser: primero, los de mala clasificación, es decir, en el que un caso puede ser escogido como control o viceversa. Para evitar este tipo de sesgo, se desarrollaron criterios de inclusión y exclusión claros para su diferenciación. El segundo sesgo que puede estar presente es el de medición, en el cual los investigadores hacen más detallada la búsqueda de información en alguno de los dos grupos; para corregirlo se utiliza una ficha de recolección de datos la cual es aplicada por dos investigadores. El tercer sesgo es el de selección, que se produce cuando la inclusión de los casos o los controles depende, en cierta medida, de la exposición de interés. Para evitar esto se seleccionaron los controles por medio de un muestreo probabilístico aleatorio simple. El cuarto sesgo corresponde a la confusión, el cual fue controlado desde el análisis de los datos por medio de un método de regresión logística binaria. Por último, el sesgo de vacíos en el registro de la información, en el que los datos son obtenidos de los registros de las historias clínicas con la posibilidad de que la información recolectada esté incompleta.

\section{Resultados}

Entre el primero de enero de 2013 y el treinta de junio de 2016 ingresaron al servicio de urgencias de la Clínica Especializada Los Andes, 11.768 personas, según la base de datos de esta institución (con el programa visual Fox pro versión 9.0). El universo de este estudio son aquellos pacientes con edades comprendidas entre 50 y 90 años, correspondiente a 3.175 personas. Se identificaron 97 casos de ataque cerebrovascular isquémico por medio de los códigos CIE10 y también 3.078 participantes que no tenían ataque cerebrovascular isquémico. Se realizó un muestreo probabilístico aleatorio simple para la selección de los 291 controles y se tuvieron en cuenta los criterios de inclusión y exclusión así: 5 controles no cumplieron con los criterios ( 2 ingresaron por consulta externa y 3 presentaron ataque cerebrovascular hemorrágicos); por esta razón se realizó una nueva selección aleatoria para la elección de los 5 controles faltantes, de este modo se alcanzó el tamaño muestral sin presentar pérdidas. En la figura 1 se resume el proceso para la selección de casos y controles.

En el grupo caso se encontraron 97 individuos con ataque cerebrovascular isquémico, el 72,2\% (70 casos) presentó infarto cerebral y el $27,8 \%$ (27 casos) accidente isquémico transitorio; en el grupo control el $100 \%$ (291 controles) no presentó ataque cerebrovascular isquémico.

\section{Análisis univariado en variables cuantitativas}

En el grupo caso la media de edad fue de 73,7 años con una desviación estándar (DE) de 10,5 años y un coeficiente de variación (CV) de 14,3\%. La media del índice de masa corporal (IMC) fue de 26,3 con DE de 4,1 y un CV de 15,7\%. La media de presión arterial sistólica (PAS) fue de $159 \mathrm{~mm} \mathrm{Hg}$ con DE de $31 \mathrm{~mm} \mathrm{Hg}$ y CV de 19,4\%. La media de presión arterial diastólica (PAD) fue de $86,7 \mathrm{~mm} \mathrm{Hg}$ con DE de 18,9 mm $\mathrm{Hg}$ y CV de $21,8 \%$.

En el grupo control la media de edad fue de 66,2 años con una DE de 11,3 años y CV de 17\%. La media del IMC fue de 24,6 con DE de 4,2 y un CV de $17 \%$. La media de PAS fue de $138 \mathrm{~mm} \mathrm{Hg}$ con DE de 26,4 mm Hg y CV de 19,1\%. La media de PAD fue de $81 \mathrm{~mm} \mathrm{Hg}$ con DE de 15,1 mm Hg y CV de $18,6 \%$. Con lo descrito se puede observar como la media de edad, presión arterial sistólica y presión arterial diastólica es mayor en el grupo caso. La variable presión arterial diastólica del grupo control fue la única que presentó heterogeneidad y las demás variables estudiadas se clasificaron como medianamente homogéneas.

\section{Análisis univariado en variables cualitativas}

En la tabla 2 se puede determinar que la frecuencia del sexo femenino, hipertensión arterial, diabetes, dislipidemia, tabaquismo, fibrilación auricular, antecedente de infarto agudo de miocardio y antecedente personal de ataque cerebrovascular isquémico es mayor en el grupo caso. Las variables con mayor diferencia respecto a la frecuencia en cada uno de los grupos son el antecedente de ataque cerebrovascular isquémico, la hipertensión arterial, la dislipidemia y la fibrilación auricular. 
Tabla 1 Definición conceptual, operativa y escala de medición de las principales variables del estudio

\begin{tabular}{|c|c|c|c|}
\hline Variable & Definición conceptual & Definición operativa & $\begin{array}{l}\text { Escala de } \\
\text { medición }\end{array}$ \\
\hline $\begin{array}{l}\text { Ataque } \\
\text { cerebro- } \\
\text { vascular } \\
\text { isquémico }\end{array}$ & $\begin{array}{l}\text { Episodio de déficit encefálico focal que } \\
\text { aparece como consecuencia de una } \\
\text { alteración circulatoria isquémica en una } \\
\text { zona del parénquima encefálico }\end{array}$ & $\begin{array}{l}\text { Paciente con diagnóstico de infarto cerebral } \\
\text { isquémico o accidente isquémico transitorio } \\
\text { en los registros de la historia clínica }\end{array}$ & $\begin{array}{l}\text { Nominal } \\
\text { dicotómica }\end{array}$ \\
\hline $\begin{array}{l}\text { Infarto } \\
\text { cerebral } \\
\text { isquémico }\end{array}$ & $\begin{array}{l}\text { Infarto del sistema nervioso central } \\
\text { acompañado de síntomas neurológicos, la } \\
\text { mayoría de las veces focales. En las } \\
\text { situaciones en las cuales no se puede } \\
\text { realizar un diagnóstico por imagen, este es } \\
\text { reconocido clínicamente por la persistencia } \\
\text { del déficit neurológico con duración mayor } \\
\text { a } 24 \text { horas }^{8}\end{array}$ & $\begin{array}{l}\text { Dato obtenido de la historia clínica que } \\
\text { evidencie déficit neurológico (afasia, } \\
\text { paresia, desviación de la comisura labial, } \\
\text { alteración del estado de conciencia) con } \\
\text { duración mayor a } 24 \text { horas y } \\
\text { neuroimagen-TAC o RNM que indique infarto } \\
\text { del sistema nervioso central }\end{array}$ & $\begin{array}{l}\text { Nominal } \\
\text { dicotómica }\end{array}$ \\
\hline $\begin{array}{l}\text { Accidente } \\
\text { isquémico } \\
\text { transito- } \\
\text { rio }\end{array}$ & $\begin{array}{l}\text { Episodio transitorio de disfunción } \\
\text { neurológica sin la presencia de infarto } \\
\text { cerebral, del cordón espinal o de la retina }\end{array}$ & $\begin{array}{l}\text { Paciente con diagnóstico definitivo de } \\
\text { accidente isquémico transitorio registrado } \\
\text { en la historia; clínicamente caracterizado } \\
\text { por afasia, paresia, desviación de la } \\
\text { comisura labial y/o alteración de } \\
\text { consciencia de carácter transitorio y con } \\
\text { neuroimagen que descarte infarto del } \\
\text { sistema nervioso central }\end{array}$ & $\begin{array}{l}\text { Nominal } \\
\text { dicotómica }\end{array}$ \\
\hline $\begin{array}{l}\text { Hipertensión } \\
\text { arterial }\end{array}$ & $\begin{array}{l}\text { Según el séptimo comité (JNC 7) se define } \\
\text { como presión arterial sistólica igual o } \\
\text { mayor a } 140 \mathrm{~mm} \mathrm{Hg} \text { y/o presión arterial } \\
\text { diastólica igual o mayor a } 90 \mathrm{~mm} \mathrm{Hg}^{9}\end{array}$ & $\begin{array}{l}\text { Paciente con cualesquiera de los siguientes } \\
\text { tres criterios en los registros de historia } \\
\text { clínica: diagnóstico de HTA según el JNC } 7 \text { o } \\
\text { antecedente de HTA y/o que reciba } \\
\text { medicación antihipertensiva en los últimos } \\
\text { seis meses }\end{array}$ & $\begin{array}{l}\text { Nominal } \\
\text { dicotómica }\end{array}$ \\
\hline $\begin{array}{l}\text { Diabetes } \\
\text { mellitus }\end{array}$ & $\begin{array}{l}\text { Desorden metabólico de múltiples } \\
\text { etiologías, caracterizado por hiperglucemia } \\
\text { crónica con disturbios en el metabolismo de } \\
\text { los carbohidratos, grasas y proteínas y que } \\
\text { resulta de defectos en la secreción y/o en } \\
\text { la acción de la insulina }{ }^{10}\end{array}$ & $\begin{array}{l}\text { Registro en la histórica clínica de } \\
\text { antecedente de diabetes mellitus, uso de } \\
\text { antidiabético oral y/o insulina en los } \\
\text { últimos seis meses o alguno de los } \\
\text { siguientes criterios diagnósticos: } \\
\text { Glicemia en ayunas igual o mayor a } \\
126 \mathrm{mg} / \mathrm{dl} \\
\text { Glicemia igual o mayor a } 200 \mathrm{mg} / \mathrm{dl} \text {, medida } \\
\text { a las } 2 \text { horas, posterior a una carga de } \\
\text { glucosa de } 75 \mathrm{~g} \\
\text { Paciente con síntomas clásicos de diabetes } \\
\text { o de crisis hiperglucemia con glicemia al } \\
\text { azar igual o mayor a } 200 \mathrm{mg} / \mathrm{dl} \\
\text { Hemoglobina glucosilada igual o mayor a } \\
6,5 \%{ }^{11}\end{array}$ & $\begin{array}{l}\text { Nominal } \\
\text { dicotómica }\end{array}$ \\
\hline Dislipidemia & $\begin{array}{l}\text { Alteración en la función o la cantidad de } \\
\text { lipoproteínas del plasma. El nivel de cada } \\
\text { una de las metas de colesterol está } \\
\text { determinado por la clasificación de riesgo } \\
\text { cardiovascular de cada individuo }{ }^{12}\end{array}$ & $\begin{array}{l}\text { Registro en la histórica clínica del } \\
\text { antecedente de dislipidemia y/o el uso de } \\
\text { hipolipemiantes orales en los últimos seis } \\
\text { meses }\end{array}$ & $\begin{array}{l}\text { Nominal } \\
\text { dicotómica }\end{array}$ \\
\hline $\begin{array}{l}\text { Fibrilación } \\
\text { auricular }\end{array}$ & $\begin{array}{l}\text { Taquiarritmia supraventricular con } \\
\text { activación auricular no coordinada y como } \\
\text { consecuencia una contracción auricular } \\
\text { ineficaz }^{13}\end{array}$ & $\begin{array}{l}\text { Registro en la historia clínica del } \\
\text { diagnóstico electrocardiográfico de } \\
\text { fibrilación auricular (intervalo R-R irregular, } \\
\text { ausencia de ondas P y actividad auricular } \\
\text { irregular) al ingreso a urgencias o con } \\
\text { antecedente de fibrilación auricular }\end{array}$ & $\begin{array}{l}\text { Nominal } \\
\text { dicotómica }\end{array}$ \\
\hline
\end{tabular}


Tabla 1 (continuación)

\begin{tabular}{|c|c|c|c|}
\hline Variable & Definición conceptual & Definición operativa & $\begin{array}{l}\text { Escala de } \\
\text { medición }\end{array}$ \\
\hline Tabaquismo & $\begin{array}{l}\text { Enfermedad adictiva crónica que } \\
\text { evoluciona con recaídas, caracterizada por } \\
\text { el consumo de tabaco }{ }^{14}\end{array}$ & $\begin{array}{l}\text { Consumo de tabaco registrado en la historia } \\
\text { clínica }\end{array}$ & $\begin{array}{l}\text { Nominal } \\
\text { dicotómica }\end{array}$ \\
\hline $\begin{array}{l}\text { Antecedente } \\
\text { de ataque } \\
\text { cerebro- } \\
\text { vascular } \\
\text { isquémico }\end{array}$ & $\begin{array}{l}\text { Antecedente de episodio de déficit } \\
\text { encefálico focal que aparece como } \\
\text { consecuencia de una alteración circulatoria } \\
\text { isquémica en una zona del parénquima } \\
\text { encefálico }\end{array}$ & $\begin{array}{l}\text { Antecedente de ACV isquémico (infarto } \\
\text { cerebral isquémico o AIT) registrado en la } \\
\text { historia clínica }\end{array}$ & $\begin{array}{l}\text { Nominal } \\
\text { dicotómica }\end{array}$ \\
\hline $\begin{array}{l}\text { Antecedente } \\
\text { de infarto } \\
\text { agudo de } \\
\text { miocardio }\end{array}$ & $\begin{array}{l}\text { Evidencia de necrosis miocárdica y un } \\
\text { ambiente clínico que concuerda con } \\
\text { isquemia miocárdica }^{15}\end{array}$ & $\begin{array}{l}\text { Antecedente de IAM registrado en la } \\
\text { historia clínica }\end{array}$ & $\begin{array}{l}\text { Nominal } \\
\text { dicotómica }\end{array}$ \\
\hline
\end{tabular}

Criterios de inclusión:

Edad entre 50 y 90 años.

Pacientes atendidos en el servicio de urgencias entre el 1 de enero de 2013 y el 30 de junio de 2016

Individuos con y sin diagnóstico de $\mathrm{ACV}$ isquémico (infarto cerebral isquémico confirmado por neuroimagen o AIT)

Criterios de exclusión

Paciente con trombosis cerebral venosa, tumores del sistema nervioso central, infecciones del sistema nervioso central, epilepsia, accidente

cerebrovascular hemorrágico o hipoglucemia.
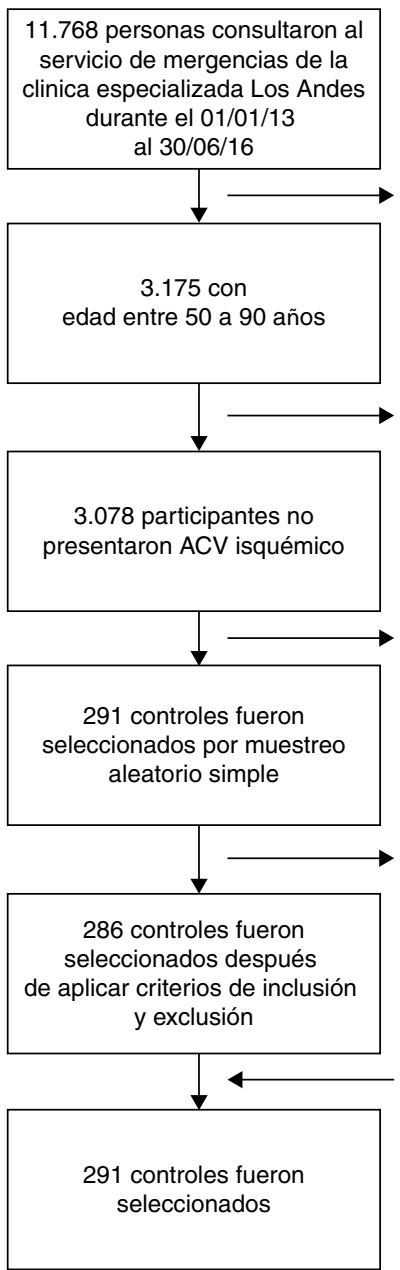

Se excluyeron 8.593 pacientes por no cumplir el criterio de edad

97 casos fueron seleccionados con ACV isquémico según los códigos $\mathrm{CIE} 10$ que cumplieron los criterios de inclusión y exclusión

Se excluyeron 2787 paciente por no ser seleccionados por el muestreo aleatorio simple

Se excluyeron 5 controles por no cumplir con los criterios de inclusión (2 ingresaron por consulta externa y 3 presentaron ACV hemorrágico)

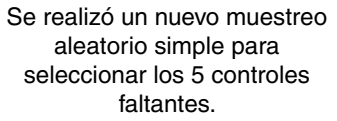
aleatorio simple para seleccionar los 5 controles faltantes.

Figura 1 Proceso para la obtención de los casos y controles según el tamaño de la muestra. 
Tabla 2 Resultado de las variables cualitativas en grupo caso y control

\begin{tabular}{|c|c|c|}
\hline Variable & Grupo caso & Grupo control \\
\hline Sexo & Femenino en el $56,7 \%$ (55 casos) & Femenino en el $54,6 \%$ (154 controles) \\
\hline HTA & $72,2 \%$ (70 casos) & $44 \%$ (128 controles) \\
\hline DM & $10,3 \%$ (10 casos) & $6,2 \%$ (18 controles) \\
\hline Dislipidemia & $13,4 \%$ (13 casos) & $4,1 \%$ ( 12 controles) \\
\hline Tabaquismo & $4,2 \%$ (4 casos) & $2,1 \%$ (6 controles) \\
\hline Fibrilación auricular & $8,2 \%$ (8 casos) & $1,7 \%$ (5 controles) \\
\hline Antecedente de IAM & $4,1 \%$ (4 casos) & 2,4\% (7 controles) \\
\hline Antecedente de $\mathrm{ACV}$ isquémico & $24,7 \%$ (24 casos) & $3,4 \%$ (10 controles) \\
\hline
\end{tabular}

HTA: hipertensión arterial, DM: diabetes mellitus, IAM: infarto agudo de miocardio, ACV: ataque cerebrovascular.

\section{Comparación de todas las variables estudiadas entre grupo caso y control}

En la tabla 3 , en relación a las variables cualitativas, se observa diferencia estadísticamente significativa entre el grupo caso y control respecto a hipertensión arterial, dislipidemia, fibrilación auricular, antecedente de infarto agudo de miocardio y antecedente de ataque cerebrovascular isquémico. No existe diferencia en relación a la variable sexo. Sin embargo, cuando se comparan las variables de tipo cuantitativo se presenta diferencia en la edad, la presión arterial diastólica y la presión arterial sistólica.

\section{Análisis bivariado de los factores asociados al ataque cerebrovascular isquémico}

En la tabla 4 se presentan los resultados del análisis bivariado con la obtención del OR crudo, su IC al 95\% y el valor $\mathrm{p}$ respectivo. En orden de importancia se demostró la asociación probable como factor de riesgo para ataque cerebrovascular isquémico: antecedente de ataque cerebrovascular isquémico, fibrilación auricular, dislipidemia, hipertensión arterial y edad igual o mayor a 70 años.

\section{Análisis multivariado}

En el análisis multivariado, controlando factores de confusión de la tabla 5 se encuentran como probables factores de riesgo para ataque cerebrovascular isquémico, en orden de importancia: antecedente de ataque cerebrovascular isquémico, tabaquismo, dislipidemia, edad igual o mayor a 70 años e hipertensión arterial.

\section{Discusión}

Los participantes con ataque cerebrovascular isquémico en este estudio en la mayoría de los casos pertenecían al sexo femenino $(56,7 \%)$, hecho que concuerda con los datos recolectados por Triana $(54,7 \%)^{16}$ y Bayona $(64,8 \%)^{17}$, a

Tabla 3 Comparación de las variables estudiadas entre grupo caso y control

\begin{tabular}{|c|c|c|c|}
\hline Variable & Grupo caso & Grupo control & Valor $\mathrm{p}$ \\
\hline Sexo & Femenino en el $56,7 \%$ (55 casos) & $\begin{array}{l}\text { Femenino en el } 54,6 \% \text { (154 } \\
\text { controles) }\end{array}$ & $0,069^{a}$ \\
\hline Edad & Media: 73,7 años DE: 10,5 años & $\begin{array}{l}\text { Media: } 64,5 \text { años } \\
\text { DE: } 11,3 \text { años }\end{array}$ & $0,000^{b}$ \\
\hline IMC & $\begin{array}{l}\text { Media: } 26,3 \text { IMC } \\
\text { DE: } 4,1\end{array}$ & $\begin{array}{l}\text { Media: } 24,6 \\
\text { DE: } 4,2\end{array}$ & $0,059^{b}$ \\
\hline PAS & $\begin{array}{l}\text { Media: } 159,2 \mathrm{~mm} \mathrm{Hg} \\
\text { DE: } 31 \mathrm{~mm} \mathrm{Hg}\end{array}$ & $\begin{array}{l}\text { Media: } 138,9 \mathrm{~mm} \mathrm{Hg} \\
\text { DE: } 26,4 \mathrm{~mm} \mathrm{Hg}\end{array}$ & $0,000^{b}$ \\
\hline PAD & $\begin{array}{l}\text { Media: } 86,7 \mathrm{~mm} \mathrm{Hg} \\
\text { DE: } 18,9 \mathrm{~mm} \mathrm{Hg}\end{array}$ & $\begin{array}{l}\text { Media: } 81 \mathrm{~mm} \mathrm{Hg} \\
\text { DE: } 15,1 \mathrm{~mm} \mathrm{Hg}\end{array}$ & $0,000^{b}$ \\
\hline Hipertensión arterial & $72,2 \%$ (70 casos) & $44 \%$ (128 controles). & $0,000^{\mathrm{a}}$ \\
\hline Diabetes mellitus & $10,3 \%$ (10 casos) & 6,2\% (18 controles) & $0,174^{a}$ \\
\hline Dislipidemia & $13,4 \%$ (13 casos) & $4,1 \%$ ( 12 controles) & $0,001^{a}$ \\
\hline Tabaquismo & $4,2 \%$ (4 casos) & $2,1 \%$ (6 controles) & $0,260^{a}$ \\
\hline Fibrilación auricular & $8,2 \%$ (8 casos) & $1,7 \%$ (5 controles) & $0,002^{\mathrm{a}}$ \\
\hline Antecedente de IAM & $4,1 \%$ (4 casos) & $2,4 \%$ (7 controles) & $0,368^{a}$ \\
\hline Antecedente de $\mathrm{ACV}$ isquémico & $24,7 \%$ ( 24 casos) & $3,4 \%$ (10 controles) & $0,000^{\mathrm{a}}$ \\
\hline
\end{tabular}

a Con prueba de $\mathrm{Chi}^{2}$, bCon prueba no paramétrica; U de Mann-Whitney, IMC: índice de masa corporal, PAS: presión arterial sistólica, PAD: presión arterial diastólica. IAM: infarto agudo de miocardio y ACV: ataque cerebrovascular. 
Tabla 4 Análisis bivariado de los factores asociados al accidente cerebrovascular isquémico

\begin{tabular}{|c|c|c|c|c|c|}
\hline Variable & $\begin{array}{l}\text { Frecuencias en } \\
\text { el grupo caso }\end{array}$ & $\begin{array}{l}\text { Frecuencias en el } \\
\text { grupo control }\end{array}$ & OR & IC $95 \%$ & Valor $\mathrm{p}$ \\
\hline Antecedente & $24,7 \%$ ( 24 casos) & $3,4 \%$ (10 controles) & & & \\
\hline $\begin{array}{l}\text { personal de ACV } \\
\text { isquémico }\end{array}$ & & & 9,3 & 4,$28 ; 20,47$ & 0,000 \\
\hline Fibrilación auricular & $8,2 \%$ (8 casos) & $1,7 \%$ (5 controles) & 5,2 & 1,$65 ; 16,30$ & 0,002 \\
\hline Dislipidemia & $13,4 \%$ (13 casos) & $4,1 \%$ (12 controles) & 3,5 & 1,$58 ; 8,18$ & 0,001 \\
\hline HTA & $72,2 \%$ (70 casos) & $44 \%$ (128 controles) & 3,3 & 2,$00 ; 5,40$ & 0,000 \\
\hline $\begin{array}{l}\text { Edad igual o mayor a } \\
70 \text { años }\end{array}$ & $67 \%$ (65 casos) & $\begin{array}{l}39,7 \%(115 \\
\text { controles) }\end{array}$ & 3,0 & 1,$90 ; 5,01$ & 0,000 \\
\hline Tabaquismo & $4,2 \%$ (4 casos) & $2,1 \%$ (6 controles) & 2,0 & 0,$57 ; 7,47$ & 0,260 \\
\hline Antecedente de IAM & $4,1 \%$ (4 casos) & $2,4 \%$ (7 controles) & 1,7 & 0,$50 ; 6,16$ & 0,368 \\
\hline $\mathrm{DM}$ & $10,3 \%$ (10 casos) & $6,2 \%$ (18 controles) & 1,7 & 0,$77 ; 3,91$ & 0,174 \\
\hline Sexo & $\begin{array}{l}\text { Masculino en el } \\
43,2 \% \text { ( } 42 \text { casos) }\end{array}$ & $\begin{array}{l}\text { Masculino en el } 47 \% \\
\text { (137 controles) }\end{array}$ & 0,9 & 0,$57 ; 1,45$ & 0,698 \\
\hline
\end{tabular}

OR: odds ratio, IC: intervalo de confianza, ACV: ataque cerebrovascular, HTA: hipertensión arterial, IAM: infarto agudo de miocardio, DM: diabetes mellitus.

diferencia de Bonilla y Aldoaneth, en cuyos estudios predominó el sexo masculino con $50,4 \%{ }^{18}$ y $60,4 \%{ }^{19}$. La media de la edad en el grupo de ataque cerebrovascular isquémico fue de 73,7 años, mayor que lo encontrado en el estudio de Pérez $(67,8 \text { años para hombres y } 67,9 \text { años para mujeres })^{20}$. En relación a los factores asociados, el antecedente de ataque cerebrovascular isquémico mostró mayor asociación como factor de riesgo (OR 7,7), superando los resultados de Hervas $(\mathrm{OR} 4)^{21}$. El tabaquismo también presentó asociación (OR $4,4)$ y ésta fue mayor que la reportada por Porras $(\text { OR } 1,5)^{22}$. La dislipidemia (OR 3) es otro factor asociado, el cual también fue registrado en los estudios de Moreno (OR 2,6 ${ }^{23}$ y Rubeaan (OR 1,3) ${ }^{24}$. La edad igual o mayor a 70 años se asocia al ataque cerebrovascular isquémico (OR 2,3); la hipertensión arterial presentó asociación para la aparición de ataque cerebrovascular isquémico $(\mathrm{OR} 1,8)$ pero con menor fuerza que los datos obtenidos por Moreno $\left(\right.$ OR 2,8) ${ }^{23}$ y Sanossian $(\mathrm{OR} 2,6)^{25}$. La fibrilación auricular es una arritmia reconocida como un factor de riesgo para ACV, pero en este estudio después del análisis multivariado no presentó asociación; esto podría sugerir que en la población de esta investigación la frecuencia en la presentación de la fibrilación auricular no fue lo suficientemente alta como para hallar diferencias estadísticas respecto a los controles y/o también que para esta población no es un factor determinante en la presentación de la enfermedad.

Se cumplió el objetivo principal de esta investigación el cual era determinar los factores asociados al ataque cerebrovascular isquémico y se encontró, en orden de importancia: antecedente de ataque cerebrovascular isquémico (OR 7,7), tabaquismo (OR 4,4), dislipidemia (OR 3,0), edad igual o mayor a 70 años (OR 2,3) e hipertensión arterial $(O R 1,8)$. Estos datos presentan una validez interna adecuada para ser tenidos en cuenta en el servicio de urgencias de la Clínica Especializada Los Andes. En cuanto a la validez externa, podrían generalizarse a otros servicios de urgencias de la ciudad de Tunja si los pacientes cumplieran los criterios de edad y de diagnóstico del ataque cerebrovascular isquémico. Además, es prudente decir, que a pesar de intentar controlar todos los posibles sesgos en esta investigación, el tipo de diseño de estudio, la recolección retrospectiva de los datos y la falta en el registro de la información pueden afectar los resultados. Se espera que este trabajo sea la base para futuras investigaciones, que idealmente deberían ser

Tabla 5 Análisis multivariado con modelo de regresión logística binaria

\begin{tabular}{llll}
\hline Variable & OR ajustado & IC 95\% & Valor $\mathrm{p}$ \\
\hline Antecedente de ACV isquémico & 7,71 & 3,$29 ; 18,08$ & 0,000 \\
Tabaquismo & 4,48 & 1,$11 ; 18,02$ & 0,022 \\
Dislipidemia & 3,04 & 1,$22 ; 7,57$ & 0,017 \\
Edad igual o mayor a 70 años & 2,36 & 1,$36 ; 4,10$ & 0,002 \\
HTA & 1,88 & 1,$06 ; 3,33$ & 0,029 \\
Fibrilación auricular & 2,67 & 0,$74 ; 9,59$ & 0,130 \\
DM & 1,46 & 0,$56 ; 3,82$ & 0,436 \\
Antecedente de IAM & 0,76 & 0,$17 ; 3,35$ & 0,722 \\
Sexo masculino & 0,71 & 0,$41 ; 1,23$ & 0,235 \\
\hline
\end{tabular}

OR: odds ratio, IC: intervalo de confianza, HTA: hipertensión arterial, DM: diabetes mellitus. IAM: infarto agudo de miocardio, ACV: ataque cerebrovascular. 
hechas con un diseño epidemiológico de mayor jerarquía, como un estudio de cohorte.

\section{Conclusión}

Los factores que se asociaron al ataque cerebrovascular isquémico en el servicio de urgencias de la Clínica Especializada Los Andes entre los años 2013 a 2016, son, en orden de importancia: antecedente de ataque cerebrovascular isquémico, tabaquismo, dislipidemia, edad igual o mayor a 70 años e hipertensión arterial.

\section{Responsabilidades éticas}

Protección de personas y animales. Los autores declaran que para esta investigación no se han realizado experimentos en seres humanos ni en animales.

Confidencialidad de los datos. Los autores declaran que en este artículo no aparecen datos de pacientes.

Derecho a la privacidad y consentimiento informado. Los autores declaran que en este artículo no aparecen datos de pacientes.

\section{Financiación}

Ninguna.

\section{Conflictos de interés}

Ninguno.

\section{Bibliografía}

1. Mukherjee D, Patil CG. Epidemiology and the global burden of stroke. World Neurosurg. 2011;76:s85-90.

2. Santalucia P, Baviera M, Cortesi I, Tettamanti M, Marzona I, Nobili A, et al. Epidemiologic trends in hospitalized ischemic stroke from 2002 to 2010: results from a large italian population-based study. J Stroke Cerebrovasc Dis. 2015;24: 1917-23.

3. Fernando I, Pamela S, Alejandra I. Cardiovascular disease in latinamerica: the growing epidemic. Prog Cardiovasc Dis. 2014;57: 262-7.

4. Silva F, Quintero C, Zarruk JG. Comportamiento epidemiológico de la enfermedad cerebrovascular en la población colombiana. Asociación colombiana de neurología [Serial online]. [Acceso Nov 2008]. Disponible en: http://www.acnweb.org/guia/ g8cap2.pdf.

5. Lavados PM, Hennis AJ, Fernandes JG, Medina MT, Legetic B, Hoppe A, et al. Stroke epidemiology, prevention, and management strategies at a regional level: Latin America and the Caribbean. Lancet Neurol. 2007;6:362-72.

6. Frómeta Guerra A, Alvarez Aliaga A, Sánchez Figueredo SA, Fonseca Muñoz JC, Quesada Vázquez A. Factores de riesgos de la enfermedad cerebrovascular aguda. Revista Habanera de Ciencias Médicas. 2010;9:534-44.

7. Alvarez Sabín J. Patología cerebrovascular. Manual de terapéutica médica. Barcelona: Editorial Masson; 2002. p. 527-41.
8. Kernan WN, Ovbiagele B, Black HR, Bravata DM, Chimowitz MI, Ezekowitz MD, et al. Guidelines for the prevention of stroke in patients with stroke and transient ischemic attack a guideline for healthcare professionals from the American Heart Association/American Stroke Association. Stroke. 2014;45: 2160-236.

9. Chobanian AV, Bakris GL, black HR, Cushman WC, Green LA, Izzo $\mathrm{JL}$, et al. Seventh report of the joint national committee on prevention, detection, evaluation, and treatment of high blood pressure. Hypertension. 2003;42:1206-52.

10. De diabetes al. Guías ALAD de diagnóstico, control y tratamiento de diabetes mellitus tipo 2 [internet]. Washington, 2008. Disponible en: http://www2.paho.org/hq/index.php?option= com_content\&view=article\&tid=2164\%3A2010-guias-aladdiagnostico-control-tratamiento-diabetes-mellitus-tipo-2\& catid $=911 \% 3$ Adiabetes-contents\&ltemid=40306\&lang=es.

11. American diabetes association standards of medical care in diabetes. Diabetes Care. 2016; 39(39)(suppl. 1):s1-s2.

12. Reiner Z, Catapano AL, De Backer G, Graham I, Taskinen MR, Wiklund $\mathrm{O}$, et al. ESC/EAS guidelines for the management of dyslipidaemias. Eur Heart J. 2011;32:1769-818.

13. January CT, Wann LS, Alpert JS, Calkins H, Cigarroa JE, Conti JB, et al. AHA/ACC/HRS Guideline for the management of patients with atrial fibrillation: a report of the American College Of Cardiology/American Heart Association Task Force On Practice Guidelines And The Heart Rhythm Society. J Am Coll Cardiol. 2014;64:e1-76.

14. Ascanio S, Barrenechea C, De León M, García T, Gómez E, Gonzalez G. Manual nacional de abordaje del tabaquismo: en el primer nivel de atención. [Internet]. OPS. OMS. PRONACCAN. Disponible en: http://www. Comepa. Com. Uy/escuela/ pluginfile. Php/666/mod_resource/content/1/manualpna_2009. Pdf.

15. Thygesen K, Aalpert JS, Jaffe AS, Simoons ML, Chaitman BR, White HD. Third universal definition of myocardial infarction. Circulation. 2012;126:2020-35.

16. Triana JD, Becerra GP. Tiempo puerta-TAC antes y después de la implementación de una vía clínica para la atención de los pacientes que ingresan con diagnóstico de ataque cerebrovascular isquémico hiperagudo al servicio de urgencias del hospital de San José. Acta Neurol Colomb. 31(2):134-40.

17. Bayona O, Hernán F, Martínez CF, Guio CM, Díaz C, Andrés C. Utilidad del código de ataque cerebrovascular en pacientes con accidente cerebrovascular agudo: comparación de dos períodos de tiempo. Acta Neurol Colomb. 2014;30:256-62.

18. Bonilla S, Patricia N, Oliveros H, Proaños J, Espinel B, Álvarez JC, et al. Estudio de frecuencia de los factores de riesgo asociados al desarrollo de enfermedad cerebrovascular isquémica no embólica en un hospital de tercer nivel. Acta Neurol Colomb. 2014;30:149-55.

19. Hernández I, Victoria A. Caracterización epidemiológica de la enfermedad cerebrovascular isquémica en pacientes del área de emergencia ciudad hospitalaria Dr. Enrique Tejera. 2014 [Internet] Disponible en: http://www.riuc.bc.uc.edu. ve/handle/123456789/501.

20. Carreño JG, Aristizábal IC, Franco AL. Factores de riesgo relacionados con la mortalidad por enfermedad cerebrovascular, Armenia, Colombia, 2008. latreia. 2011;24:26-33.

21. Hervas A. [stroke risk factors. Population case-control study in the foral community of Navarre]. Anales del Sstema Ssanitario de Navarra. 2004;28:325-34.

22. Porras AC. Ausencia de asociación entre obesidad y sobrepeso con enfermedad cerebrovascular isquémica aguda en una muestra de población colombiana. Acta Neurol Colomb. 2010; 26.

23. Moreno C. Prevalence of stroke and associated risk factors in older adults in havana city and Matanzas provinces, Cuba (10/66 population-based study). Medicc Review. 2010;12:20. 
24. Al-Rubeaan K, Al-Hussain F, Youssef AM, Subhani SN, Al-Sharqawi $\mathrm{AH}$, Ibrahim HM. Ischemic stroke and its risk factors in a registrybased large cross-sectional diabetic cohort in a country facing a diabetes epidemic. J Diabetes Res. 2016.
25. Sanossian N, Azen SP, Varma R. Prevalence and risk factors for cerebrovascular disease in communitydwelling latinos. Clin Neurol Neurosur. 2008;110: 985-7. 